\title{
HIV epidemiology and responses among men who have sex with men and transgender individuals in China: a scoping review
}

Songyuan Tang ${ }^{1,2,3,4+}$, Weiming Tang ${ }^{1,2,3 \dagger}{ }^{+}$Kathrine Meyers ${ }^{5}$, Polin Chan ${ }^{6}$, Zhongdan Chen $^{7}$ and Joseph D. Tucker ${ }^{1,2,3^{*}}$

\begin{abstract}
Background: Despite global efforts to control HIV among key populations, new infections among men who have sex with men (MSM) and transgender (TG) individuals are still increasing. The increasing HIV epidemic among MSM/TG in China indicates that more effective services are urgently needed. However, policymakers and program managers must have a clear understanding of MSM/TG sexual health in China to improve service delivery. To meet this need, we undertook a scoping review to summarize HIV epidemiology and responses among MSM and TG individuals in China.

Methods: We searched MEDLINE, EMBASE and the Cochrane Library for recent studies on MSM/TG HIV epidemiology and responses. We also included supplemental articles, grey literature, government reports, policy documents, and best practice guidelines.

Results: Overall, HIV prevalence among Chinese MSM was approximately $8 \%$ in 2015 with a higher prevalence observed in Southwest China. TG are not captured in national HIV, STD, or other sexual health surveillance systems. There is limited data sharing between the public health authorities and community-based organizations (CBOs). Like other low and middle income countries, China is challenged by low rates of HIV testing, linkage, and retention. Several pilot interventions have been shown to be effective to increase HIV testing among MSM and TG individuals, but have not been widely scaled up. Data from two randomized controlled trials suggests that crowdsourcing contests can increase HIV testing, creating demand for services while engaging communities.

Conclusion: Improving HIV surveillance and expanding HIV interventions for Chinese MSM and TG individuals are essential. Further implementation research is needed to ensure high-quality HIV services for MSM and TG individuals in China.
\end{abstract}

Keywords: HIV epidemics and responses, Men who have sex with men, HIV care continuum, Scoping review, China

\footnotetext{
* Correspondence: jdtucker@med.unc.edu

Songyuan Tang and Weiming Tang are co-first authors.

${ }^{\dagger}$ Equal contributors

'University of North Carolina Project-China, No. 2 Lujing Road, Guangzhou

510095, China

${ }^{2}$ Guangdong Provincial Center for Skin Diseases and STI Control, Guangzhou,

China

Full list of author information is available at the end of the article
} 


\section{Background}

In 2015 the WHO launched a comprehensive revision of the 2013 consolidated ARV guidelines, recommending immediate treatment for all people living with HIV and pre-exposure prophylaxis (PrEP) for people at high risk of HIV infection [1, 2]. At the same time, UNAIDS declared that the world should end the AIDS epidemic by 2030, with the goal of achieving the 90-90-90 diagnosis and treatment targets by 2020 [3]. Given these goals, it is imperative that we have a better understanding of key populations, including men who have sex with men (MSM) and transgender individuals (TG).

Globally, MSM are 19 times more likely to be living with HIV than the general population [4]. Despite global efforts to control HIV among key populations, new infections among MSM are still increasing [5-7]. High HIV prevalence was consistently observed among MSM in many regions around the world. For example in 2012, the HIV prevalence among MSM in the Caribbean was as high as $25 \%$ [8]. Similar patterns have also been observed in Asia [8]. TG individuals are also an important, but often neglected, key population $[9,10]$.

The Chinese government has increased policy attention to HIV in recent years, providing an opportunity to enhance service delivery for MSM and TG individuals [11]. The purpose of this scoping review was to summarize HIV epidemiology and responses among MSM and TG individuals in China.

\section{Methods}

We undertook a scoping review to summarize the MSM/ TG HIV epidemics in China, sexual health services across the HIV care continuum, and interventions for Chinese MSM/TG. In addition, based on the literature review, we summarized several policy points for consideration.

We used Arksey and O'Malley's framework for conducting this scoping study. Scoping studies summarize key evidence on a topic, but do not go through the process of a formal systematic review [12]. We identified studies published between January 2009 and October 2015 that reported on Chinese MSM HIV epidemiology, sexual health services across the HIV care continuum, and sexual health interventions. Studies were identified using keyword searches in electronic databases. We searched the following databases: MEDLINE (OVID interface, 1946 onwards), EMBASE (OVID interface, 1947 onwards) and the Cochrane Library. The search string used synonyms and variations of the following terms: MSM, TG, meta-analysis, review and China.

We included studies that had the following elements: 1) Study designs were systematic reviews; 2) Study participants were MSM or TG in China; and 3) Outcomes included data on health services across the HIV care continuum or interventions targeting Chinese MSM or
TG. If data of interest were not available in systematic reviews, supplemental articles, grey literature, government reports, policy documents, and best practice guidelines were also included. We also contacted experts at the Chinese CDC, WHO, Gates Foundation and UNAIDS to provide reports, policy documents, and guidelines. Our preliminary report was reviewed by civil society organizations, UN organizations, the WHO China office, and two external reviewers.

\section{Results \\ HIV epidemiology and surveillance \\ Population size estimation and data sharing}

The WHO estimated 2-10 million MSM in China in 2009 [13]. China reported that about 2-4 \% adult male population in urban areas and 1-2 \% adult male population in rural areas are MSM, and estimated that the number of MSM in China may range from 3.1 to 6.3 million in 2009 [14]. Another study in China estimated that there were 5-10 million MSM in China [15].

Collected data is shared among some public health organizations and community-based organizations (CBOs). However, most of the MSM CBOs that work closely with local CDC have limited access to MSM health surveillance and related data [16-18].

\section{HIV prevalence among Chinese MSM}

The HIV prevalence was estimated to be $6.0 \%$ in 2010 [16], and it has gradually increased to $8.0 \%$ in 2015 (Fig. 1) [19]. Regional disparity is an important feature of the HIV epidemic among Chinese MSM. Based on a systematic review, HIV prevalence is increasing across all regions of China, particularly in the southwest region, made up of five provinces [16]. The average HIV prevalence in this region was three times the overall Chinese average. In addition, municipalities and provincial capitals also have higher HIV prevalence, compared to other cities [20].

Young MSM worldwide and in Asia have increased risks of acquiring HIV and STDs [21]. In China, a study conducted across four cities reported an HIV prevalence of $6.7 \%$ among MSM between 16 and 24 years old [22].

Routine HIV surveillance in China does not capture TG individuals and most of the studies reviewed did not mention TG individuals. One study found that $11.1 \%$ of transgender individuals self-reported HIV infection [23] and another found a high burden of intimate partner violence and risky sexual behaviors [24].

\section{Condom use and drug use among Chinese MSM}

The results from six reviews [25-30] that summarized condom usage among Chinese MSM reveal that though large efforts (e.g., condom promotion in venues, peer education, and condom promotion through social media) 


\section{HIV prevalence among Chinese MSM}

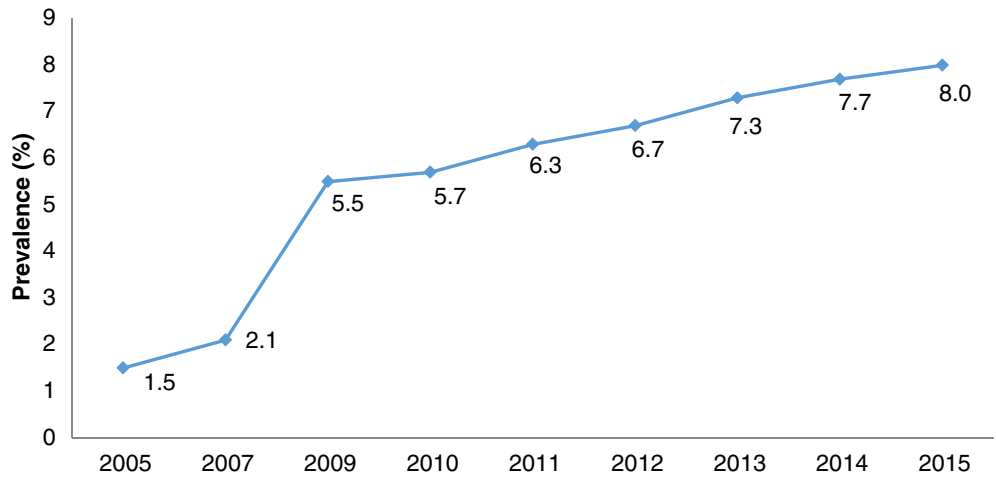

Fig. 1 HIV prevalence among Chinese MSM based on Chinese surveillance system. Source: Chinese CDC [19] and AIDSInfo (http://www.aidsinfoonline.org/)

have been made towards containing HIV, the overall rate of condomless anal intercourse (CAI) is still high. According to one meta-analysis that included 64 articles reported 82 studies [28], the CAI (during last 6 months) rate among Chinese MSM is about $54 \%$ (95 \% CI: $51-56 \%$ ). Data from the Chinese surveillance system showed that about $51 \%$ of MSM reported engaging in CAI in the last 6 months in 2013 [19]. In addition to CAI with men, many Chinese MSM also have condomless sex with women. One meta-analysis that included 43 studies reported that the pooled estimates condomless sex rate with female sex partners was $74 \%$ (95 \% CI: 72-77 \%) in the last 6 months [31].

A systematic review of 33 studies reported that the drug use rate ranged between 0.1 and $44 \%$ among Chinese MSM (median $=2.4 \%$ ) [27]. In China, recreational drug use has increased considerably among MSM in recent years [30]. A multisite cross-sectional study reported that $28 \%$ of MSM had used at least one kind of recreational drug in the last 6 months [32]. Among MSM, the most prevalent stimulant drugs were ecstasy and crystal meth. Popular non-stimulant drugs include Viagra and Ketamine [33].

\section{HIV care continuum}

Since 2008, the China MOH carried out comprehensive AIDS responses with MSM populations in 61 cities across China, including expanding MSM surveillance, increasing HIV testing, case finding, and promoting linkage to care [20]. These policies helped to define and strengthen the HIV care continuum for MSM. Programs for HIV testing, linkage to care, adherence, and retention have all expanded in the past 5 years.

\section{HIV testing}

Data from national HIV surveillance system illustrated that the HIV testing rate (tested in last year) among
Chinese MSM ranged between 43.2 and 49.0 \% [19]. In addition, results from a meta-analysis of 18 studies suggested that only $47 \%$ of Chinese MSM had ever tested for HIV, and $38 \%$ had tested during the past 12 months [34]. The number of surveys among young MSM indicated that HIV testing rates in this group were less than $30 \%$ [35]. In addition, among MSM tested for HIV, several studies suggested that HIV testing is infrequent [35-37]. One study noted poor HIV test uptake among TG individuals in China [23].

Several research studies have identified barriers and facilitators of HIV testing among MSM. Fear of stigma and discrimination, concerns about confidentiality, limited HIV knowledge, and institutional barriers such as inconvenient testing times were identified as barriers to accessing HIV testing [17, 38-40]. Practicing high risk sexual behaviors, family and partner support, rapid testing, and self-testing were mentioned as facilitators of testing $[17,38-40]$.

\section{HIV linkage to care, ARV adherence and retention in care}

Nationwide scale-up of HIV treatment programs has been implemented in China over the last decade. However, late diagnosis, incomplete linkage to care, and high rates of loss-to-follow-up remain major clinical and public health challenges in China [41]. Infrequent viral load monitoring and incomplete reporting within surveillance systems complicate more detailed analyses. Further strengthening of the surveillance system and implementation research are needed. A study based on the national epidemiology database reported that during 2006-2012, $21.3 \%$ of newly diagnosed MSM did not receive CD4 cell count testing within the 6-months following diagnosis [42]. A online survey study reported consistent results ( $74.6 \%$ of positive individuals saw a doctor in 6 months and $25.4 \%$ took over 6 months to be linked to care) [43]. Multistage stepwise HIV testing and treatment initiation 
procedures are major structural barriers to providing timely antiretroviral therapy [41].

A study conducted in 2014 indicated that many HIV-infected individuals are receiving ART that is sub-optimal, even after they receive definite diagnosed [44]. Among MSM patients with CD4 cell count $\leq 350$ cells $/ / \mathrm{mm} 3$, only around $60 \%$ received ART [44, 45]. A study conducted in Guangzhou reported that among 721 adults living with HIV receiving ART, 18.9 \% reported recent non-adherence (any missed ART in the past 4 weeks) and $6.8 \%$ reported treatment interruption ( 4 or more weeks of missed ART in the past year) [46].

Retention in care among MSM patients is also not optimal $[41,42,44]$. Based on a study conducted during 2003 to 2009, people living with HIV receiving ART reported that the median length of time on ART was 18.9 months, and the cumulative probability of attrition from ART initiation was $9 \%$ at 12 months and $24 \%$ at 60 months. This nationwide study showed that MSM retention rates were better than other risk groups. A retrospective cohort study conducted in Changsha reported a retention rate of $58.1 \%$ at 12 months, with no difference in retention reported between MSM and others [47].

These studies also showed that MSM face a number of individual and structural barriers across the HIV care continuum. Negative coping methods create significant problems for linking newly-diagnosed men to care and retaining them in care. Meanwhile, qualitative research indicates that nondisclosure of sexual orientation to families, absence peer social support and professional psychological counseling, lack of affordable and specialized treatment and care, homophobia and discrimination from providers may deter engagement in care $[40,48]$.

\section{Interventions ongoing and completed Prevention and behavioral interventions at individual and group-level}

Since 2005, the Chinese government has expanded its intervention efforts to MSM [49]. Various programs have been conducted to promote condom use, counseling and testing, peer education, and follow-up outreach. A meta-analysis that summarized 22 MSM intervention studies suggested that behavioral interventions have been efficacious in increasing HIV knowledge, HIV testing, and condom use, and in reducing sexual risk behaviors. However, the study also indicated that interventions have not reduced the incidence of HIV among Chinese MSM [50].

\section{Interventions for promoting HIV Self-testing (HIVST) in China}

HIVST is not illegal in China, and more than 20 HIVST kits have been approved by the Chinese Food and Drug Administration [51] and are available in China [52].
There is currently no national program of HIVST among MSM in China, however around one quarter of Chinese MSM have self-tested for HIV [52, 53]. Several pilot programs have shown that HIVST is an additional HIV testing method that could increase first-time HIV testing $[53,54]$. Many pilot interventions have been conducted to examine the efficacy of hybrid $\mathrm{CBO}$-clinic models in promoting HIV testing and linkage to care (Table 1). These interventions primarily focus on improving cooperation among CDC system, hospital and MSM CBOs in China $[46,55]$. For example, by collaborating with a

Table 1 Project IMPACT: A Model for Increasing the Collaboration between Community-based Organizations and the Public Sector in China

\section{Purpose of the program}

A pilot project "Integration Minimum package of Prevention in Accelerating Case finding and Treatment (IMPACT) was carried out in Guangzhou since 2008. The purpose of this project was to increase the collaboration between Community-based Organizations (CBOs) and the public sector in China to provide friendly HIV-related service to men who have sex with men (MSM).

\section{Methods of the program}

Guangzhou CDC and the Lingnan Partners Community Support Center worked together to design an integrated service including HIV health education, online HIV risk assessment, on-site HIV counseling and testing, partner notification, CD4 cell count testing, psychosocial care and support, and guidance on clinical treatment. This program includes three main parts: online prevention tools, online-to-offline service linkage, and one-stop service.

Online prevention tools: Two internet tools were developed: a scenario-based application and an HIV risk self-assessment system. The scenariobased application is an interactive internet application that simulates real-life HIV risk scenarios, in order to promote HIV testing and condom use. The online HIV risk self-assessment system evaluates an individualized HIV-risk score by evaluating an individual's risk profile. Based on the results, the system provides tailored guidance to promote HIV testing and behavioral change.

Online-to-offline service linkage: This component linked virtual intervention/ testing mobilization efforts to actual HIV testing, and facilitated HIV care. The online prevention tools described above were linked to an online appointment system for HIV testing. Test results were made available online by an anonymous fication system (Easy Tell ${ }^{\oplus}$ )

One-stop shops: In 'one-stop shops', public sector staff provided on-site blood sampling and testing and carried out epidemiological investigations. Meanwhile, CBO peers delivered high-quality and timely pre- and post-test counseling, psychosocial support services, guidance on retention to care and ART adherence support services.

\section{Outcomes}

The IMPACT model provides services on HIV health education, online HIV risk assessment, on-site HIV counseling and testing, partner notification, CD4 cell count testing, psychosocial care and support, and ART adherence guidance. The number of tests performed through IMPACT has increased from 1,064 in 2008 to 7,754 in 2013. Of the 999 HIV positive cases identified through this project between 2008 and 2013, linkage to care and retention in care rates of 95 and $89 \%$, respectively [58].

Lessons learned

This public sector-CBO hybrid model has not only addressed the needs of the MSM community, but has also been instrumental in reducing barriers of access to HIV services. 
local $\mathrm{CBO}$, the Guangzhou CDC built a social entrepreneurship model to promote HIVST among MSM [56]. Overall, 198 (52.1 \%) MSM purchased self-testing kits, and $192(97.0 \%)$ participants used kits and returned the testing results [57].

\section{Internet-based and social media interventions}

A few internet-based interventions have been conducted to improve accessibility to HIV services and care [55, 58, 59]. For example, the Guangzhou CDC developed two internet-based interventions: 'scenario experiencing intervention application' and 'online HIV risk self-assessment system' [58]. Compared to the control group, participants in the intervention arm of HIV risk-assessment system had reduced CAI in the last anal sex by $44 \%$, and CAI with regular and casual partners within the last three months by 26 and $25 \%$ respectively [58].

Various social media techniques have been applied to HIV educational interventions as well as to testing mobilization and partner services interventions targeted towards MSM in China. Danlan Gongyi, an LGBT online web portal and $\mathrm{CBO}$, used Wechat to provide rapid and anonymous HIV testing services as well as offline services such as psychological counseling and support [3]. Other social media platforms, such as QQ, Jack'd and microblogging, have been also used in carrying out HIV/AIDS interventions among Chinese MSM [60, 61].

\section{Crowdsourcing HIV test promotion interventions}

Crowdsourcing is "the practice of obtaining needed services, ideas, or content by soliciting contributions from a large group of people and especially from the online community [62]." Crowdsourcing can be used to solicit concepts, images, and videos to promote HIV testing (Table 2). Several crowdsourcing contests have been implemented in South China. One randomized controlled trial (RCT) in China aimed at promoting HIV testing showed that crowdsourcing methods were both effective and cost saving when compared to a health marketing intervention. HIV test uptake was similar between the crowdsourced arm $(37 \%, 114 / 307)$ and the health marketing arm $(35 \%, 111 / 317)$ [52]. Another RCT in China found that a crowdsourced intervention was nonequivalent to a social marketing intervention, also noting cost savings [63].

\section{Interventions for improving linkage and retention in care}

In 2012, the China CDC initiated a pilot intervention project to demonstrate a 'one-stop' service model for individuals with HIV infection [44]. This model included referral, transportation, information and emotional support. Patients in the service clinic receive all procedures and protocols in a single setting. The pilot intervention demonstrated that this model outperformed the existing
Table 2 SESH and crowdsourcing contests to create demand for HIV testing and other services

Purpose of the Program

To create more engaging and effective sexual health services using crowdsourcing and other social entrepreneurship tools.

Methods of the program

SESH has organized and evaluated several creative contributory contests and other programs [76]. For example, in 2013, we launched our first contest to solicit videos promoting HIV testing. First, SESH posted an online open call for videos and hosted a call to increase awareness of the contest. Second, a group of multisectoral judges evaluated each of the video entries, giving them a score of 1-10 and selecting a group of finalists and a single winner. Judging criteria included reaching individuals who had never tested before, generating excitement, and community responsiveness. Finally, the winner from the contest was formally evaluated and selected as an intervention tool.

In 2014, SESH launched a sexual health image contest to encourage young students to talk about sexual health. We promoted the contest information as well as sexual health knowledge among young people though online (broadcasting and interacting on wechat and weibo) and in-person events (lectures, activities, and workshops). In 2015, SESH launched a condom video contest to solicit videos promoting condom use among MSM. Then in 2016 the World Health Organization invited SESH to organize a global contest to solicit hepatitis testing innovations [73].

Outcomes

SESH has data from RCTs, qualitative research, and social media suggesting the effectiveness of crowdsourcing as an approach. Two RCTs demonstrated that crowdsourcing was effective and saved money compared to conventional evidence-based marketing approaches [52, 63].

Lesson learned

Crowdsourcing could be a useful way to spur creative, new ideas for improving health and engaging communities. This new tool may be especially useful in low and middle-income countries where civil society organizations are often constrained or less able to directly inform public health programs. Crowdsourcing contests may help create more engaging, effective, and creative campaigns [33, 35].

service model on five out of six indicators. Between 2012 and 2014, the Chinese CDC in Guangxi also piloted a program for immediate initiation of ART [41]. The intervention significantly increased ART coverage within 90 days of diagnosis of HIV infection, and reduced overall mortality from about $26 \%$ to less than $10 \%$ [41].

\section{Pre-exposure prophylaxis (PrEP)}

The use of PrEP is now recommended by the WHO for all individuals at substantial risk of acquiring HIV [64]. China has yet to make new recommendations with regard to PrEP. Few studies suggest that while awareness remains low (ranging from 2.8 to $22 \%$ ), willingness to use PrEP is high (ranging from 64.0 to $84.6 \%$ ) [65-68].

Two PrEP demonstration projects in China have been published to date $[69,70]$. The first one compared the effect of combination regimen of TDF + FTC daily to TDF + FTC taken twice weekly plus an additional dose $2 \mathrm{~h}$ before sex. Among 153 MSM, $79.7 \%$ completed 28 weeks of follow up, and $68.6 \%$ reported not missing a dose [69]. The second study allowed participants to 
choose the no drug intervention, daily TDF or pericoital dosing (1 dose TDF 24-48 h before sex plus 1 dose TDF within $2 \mathrm{~h}$ after sex). Retention at 12 months was relatively low (43.1\%), and varied significantly across study arms [70].

\section{Discussion}

Based on the epidemiology and response data, we identified a number of opportunities to strengthen the HIV response and decrease HIV risk among MSM in China. These opportunites include improving HIV surveillance and expanding interventions among MSM and transgender individuals.

\section{Improving HIV surveillance}

Given the size and complexity of China, an accurate MSM/TG HIV surveillance system is critical. Two areas could improve HIV surveillance among these key populations: 1) enhancing data sharing between $\mathrm{CBO}$ and CDCs; 2) using online surveys to capture MSM and TG individuals. Several interventions suggest the importance of collaborative $\mathrm{CBO}-\mathrm{CDC}$ programs across the continuum of care. However, MSM CBOs often have limited access to MSM sexual health surveillance data. Increased data sharing between $\mathrm{CBO}$ and local CDCs could improve service provision, needs assessment, and intervention development.

Online surveys can also help capture real-time HIV surveillance data. Large numbers of MSM in China routinely use the Internet [20], especially smart-phone based partner-seeking mobile applications [49]. Some online MSM are difficult to reach through the current MSM surveillance system [23]. Integrating online data collection into the surveillance system could make it easier to reach some subgroups of MSM. Currently, there is very limited data specific on TG individuals. Conducting online surveillance among online TG individuals could be useful, as previous studies have shown that online sampling can reduce some of the social and structural barriers that limit efforts to reach TG [23, 24]. Data security and confidentiality are important concerns in the context of using online surveys.

\section{Expanding HIV interventions for MSM and TG individuals}

Our review identified a number of MSM/TG interventions that could be expanded, including the following: 1) HIVST interventions to expand HIV testing; 2) crowdsourcing and social media interventions to stimulate demand for HIV services; 3) community-based interventions.

HIVST may be a useful tool for expanding HIV testing and promoting people-centered systems. Although HIVST systems tailored for MSM have been piloted in many Chinese cities $[53,57,58]$, none have been widely scaled up. Expanding the use of HIVST among MSM represents a major opportunity for public health systems to reach MSM and TG individuals with suboptimal HIV test uptake. The expansion of HIVST may require new organizations such as a social enterprise [71] which combine elements of enterprise and social welfare organization.

Both crowdsourcing and social media interventions have proven effective in stimulating demand for HIV services in China. Two randomized controlled trial studies demonstrated that a crowdsourcing approach was effective and cost saving method among MSM and TG individuals in China [52, 63]. This method could increase community engagement in HIV campaigns [52]. A crowdsourcing approach could also be used to increase demand for PrEP and partner services. In addition to crowdsourcing, other types of social media interventions may help to increase HIV service demand [72, 73].

Community-based interventions are an important part of the HIV response among MSM and TG individuals. Supporting local community-based organizations to implement community-based interventions should be considered. At a national and sub-national level, the participation of key populations and key populations living with HIV must be considered in formulating strategies and programming [58]. Within the many $\mathrm{CDC}-\mathrm{CBO}$ hybrid models, ensuring governance and resources to support community voices is important.

\section{Conclusion}

Implementation of HIV interventions, especially those focused on serving MSM and TG individuals, is an enormous challenge [74]. Implementation science research can help identify and anticipate problems, and in turn, answer questions on how best to deliver interventions [75]. Incomplete surveillance and reporting systems, especially for TG individuals, underlines the need for strengthened routine surveillance and further research. This scoping review suggests several unmet implementation needs for MSM and TG in China.

\section{Additional file}

Additional file 1: Annex: Summary of ongoing and completed interventions focus on the HIV care continuum among MSM in China (DOCX 137 kb)

\footnotetext{
Abbreviations

ART: Antiretroviral therapy; ARV: Antiretroviral(s); CAI: Condomless anal intercourse; CBOs: Community based organization(s); CDC: Centers for disease control and prevention; HIVST: HIV self-testing; HTS: HIV testing service; ISR: Implementation science research; KP: Key population(s); LGBT: Lesbian, gay, bisexual and transgender; MSM: Men who have sex with men; PLHIV: People living with HIV; PrEP: Pre-exposure prophylaxis; PS: Partner services; RNA: Ribonucleic acid; STDs: Sexually transmitted diseases(s); STIs: Sexually transmitted infection(s); UNAIDS: Joint United Nations Programme on HIV/AIDS; VCT: Voluntary counselling and testing; VL: Viral load; WHO: World Health Organization
} 


\section{Acknowledgement}

Sincere thanks are given to Colin Shepard, MD, and Zhijun Li, MD from Division of Global HIV/AIDS in China, China office of U.S. Centers for Disease Control \& Prevention (CDC); Dr. James Yang from UNDP and Mr. John Shen from Beijing LGBT center for their strong support during the data collection process. Thanks are also given to two excellent young scholars, Jessica Mao and Wei Zhang, for their contributions in process of literature collection and report editing. We also would like to thank the staff at Social Entrepreneurship for Sexual Health (SESH) Global, Danlan Gongyi (淡蓝公益), Lingnan Partner (岭南伙伴), Guangzhou CDC, and Guangdong Provincial Center for Skin Diseases and STI Control, who contributed to our work.

\section{Funding}

This work was supported by the WHO-China office; National Institutes of Health [National Institute of Allergy and Infectious Diseases 1R01Al114310 to JT]; and UNC-South China STD Research Training Centre [Fogarty International Centre 1D43TW009532 to JT.

\section{Availability of data and materials}

The dataset supporting the conclusions of this article is included within the article's Additional file 1.

\section{Authors' contributions}

All authors have read and approved the final manuscript. This scoping review was prepared by Drs. Songyuan Tang, Weiming Tang, Kathrine Meyers, and Joseph Tucker. Drs. Polin Chan made significant contribution in process of literature collection. Drs. Polin Chan, and Zhongdan Chen reviewed the manuscript.

\section{Competing interests}

The authors declare that they have no competing interests.

\section{Consent for publication}

Not applicable.

\section{Ethics approval and consent to participate} Not applicable.

\section{Author details}

'University of North Carolina Project-China, No. 2 Lujing Road, Guangzhou 510095, China. ${ }^{2}$ Guangdong Provincial Center for Skin Diseases and STI Control, Guangzhou, China. ${ }^{3} \mathrm{SESH}$ Global, Guangzhou, China. ${ }^{4}$ Kunming Medical University, Kunming, China. ${ }^{5}$ Aaron Diamond Aids Research Center, New York, USA. 'WHO China Office, Beijing, China. ' UNAIDS China Office, Beijing, China.

Received: 8 April 2016 Accepted: 6 October 2016

Published online: 20 October 2016

\section{References}

1. WHO. Consolidated guidelines on the use of antiretroviral drugs for treating and preventing HIV infection. Geneva: World Health Organization; 2013.

2. WHO. Guideline on when to start antiretroviral therapy and on preexposure prophylaxis for HIV. Geneva: World Health Organization; 2015.

3. UNAIDS: World AIDS Day Report: Focus on location and population. In. New York; 2015

4. UNAIDS. The Gap report, vol. 2014. New York: UNAIDS; 2014. http://files, unaids.org/en/media/unaids/contentassets/documents/unaidspublication/ 2014/UNAIDS Gap report en.pdf. Accessed 7 Oct 2016.

5. van Griensven F, van Wijngaarden JWL. A review of the epidemiology of HIV infection and prevention responses among MSM in Asia. AIDS. 2010;24:S30-40

6. van Griensven F, van Wijngaarden JWL, Baral S, Grulich A. The global epidemic of HIV infection among men who have sex with men. Curr Opin HIV AIDS. 2009:4(4):300-7.

7. Beyrer C, Baral SD, van Griensven F, Goodreau SM, Chariyalertsak S, Wirtz AL, Brookmeyer R. Global epidemiology of HIV infection in men who have sex with men. Lancet. 2012:380(9839):367-77.

8. Beyrer C, Sullivan P, Sanchez J, Baral SD, Collins C, Wirtz AL, Altman D, Trapence G, Mayer K. The increase in global HIV epidemics in MSM. AIDS. 2013;27(17):2665-78
9. Winter S, Diamond M, Green J, Karasic D, Reed T, Whittle S, Wylie K Transgender people: health at the margins of society. Lancet. 2016;16:683-8.

10. Wylie K, Knudson G, Khan S, Bionerbale M, Watanyusakul W, Baral S. Serving transgender people: clinical care considerations and service delivery models in transgender health. Lancet. 2016;16:682-6.

11. Wu Z, Wang Y. Introduction: China meets new AIDS challenges. J Acquir Immune Defic Syndr. 2010;53:S1-3.

12. Arksey H, O'Malley L. Scoping studies: towards a methodological framework. Int J Soc Res Methodol. 2005;8(1):19-32.

13. Godwin J. Legal environments, human rights and HIV responses among men who have sex with men and transgender people in Asia and the Pacific: An agenda for action. In. New York: UNDP; 2010.

14. Wang L, Wang N, Wang L, Li D, Jia M, Gao X, Qu S, Qin Q, Wang Y, Smith K. The 2007 estimates for people at risk for and living with HIV in China: progress and challenges. J Acquir Immune Defic Syndr. 2009;50(4):414-8.

15. Wong FY, Huang ZJ, Wang W, He N, Marzzurco J, Frangos S, Buchholz ME, Young D, Smith BD. STIs and HIV among men having sex with men in China: a ticking time bomb? AIDS Educ Prev. 2009;21(5):430-46.

16. Zhang L, Chow EP, Jing J, Zhuang X, Li X, He M, Sun H, Li X, Gorgens M, Wilson D. HIV prevalence in China: integration of surveillance data and a systematic review. Lancet Infect Dis. 2013;13(11):955-63.

17. Bien CH, Muessig KE, Lee R, Lo EJ, Yang LG, Yang B, Peeling RW, Tucker JD. HIV and syphilis testing preferences among men who have sex with men in South China: a qualitative analysis to inform sexual health services. PLoS One. 2015;10(4):e0124161.

18. Tucker JD, Muessig KE, Cui R, Bien CH, Lo El, Lee R, Wang K, Han L, Liu FY, Yang $L G$, et al. Organizational characteristics of HIV/syphilis testing services for men who have sex with men in South China: a social entrepreneurship analysis and implications for creating sustainable service models. BMC Infect Dis. 2014;14:601.

19. Qin Q, Tang W, Ge L, Li D, Mahapatra T, Wang L, Guo W, Cui Y, Sun J: Changing trend of HIV, Syphilis and Hepatitis C among Men Who Have Sex with Men in China. Scientific Reports 2016: In Press.

20. Wu Z, Xu J, Liu E, Mao Y, Xiao Y, Sun X, Liu Y, Jiang Y, McGoogan JM, Dou Z. HIV and syphilis prevalence among men who have sex with men: a cross-sectional survey of 61 cities in China. Clin Infect Dis. 2013:57(2):298-309.

21. Garofalo R, Herrick A, Mustanski BS, Donenberg GR. Tip of the iceberg: Young men who have sex with men, the Internet, and HIV risk. Am J Public Health. 2007;97(6):1113-7.

22. Wei S, Zhang H, Wang J, Song D, Duan Y, Yu F, She M, Wang M, Zhang H. HIV and syphilis prevalence and associated factors among young men who have sex with men in 4 cities in China. AIDS Behav. 2013;17(3):1151-8.

23. Best J, Tang W, Zhang Y, Han L, Liu F, Huang S, Yang B, Wei C, Tucker JD. Sexual Behaviors and HIV/Syphilis Testing Among Transgender Individuals in China: Implications for Expanding HIV Testing Services. Sex Transm Dis. 2015; 42(5):281-5.

24. Zhang Y, Best J, Tang W, Tso LS, Liu F, Huang S, Zheng H, Yang B, Wei C, Tucker JD: Transgender sexual health in China: a cross-sectional online survey in China. Sex Transm Infect 2016. doi: 10.1136/sextrans-2015-052350

25. Zou H, Xue H, Wang X, Lu D. Condom use in China: prevalence, policies, issues and barriers. Sex Health. 2012;9(1):27-33.

26. Zhang L, Chow EP, Wilson DP. Distributions and trends in sexual behaviors and HIV incidence among men who have sex with men in China. BMC Public Health. 2012;12(1):546.

27. Guo Y, Li X, Stanton B. HIV-related behavioral studies of men who have sex with men in China: a systematic review and recommendations for future research. AIDS Behav. 2011:15(3):521-34.

28. Wu J, Hu Y, Jia Y, Su Y, Cui H, Liu H, Wang N. Prevalence of unprotected anal intercourse among men who have sex with men in China: an updated meta-analysis. PLoS One. 2014;9(5):e98366.

29. He Q, Peng W-J, Zhang J-Q, Wang B-X, Wang J: Prevalence of unprotected anal intercourse and unprotected vaginal intercourse among HIV-positive men who have sex with men in China: a meta-analysis. Sexually transmitted infections 2011. doi: 10.1136/sextrans-2011-050230.

30. Yang Z, Zhang S, Dong Z, Jin M, Han J. Prevalence of unprotected anal intercourse in men who have sex with men recruited online versus offline: a meta-analysis. BMC Public Health. 2014;14(1):508.

31. Chow EP, Wilson DP, Zhang L. What is the potential for bisexual men in China to act as a bridge of HIV transmission to the female population? Behavioural evidence from a systematic review and meta-analysis. BMC Infect Dis. 2011;11(1):242. 
32. Xu J-J, Zhang C, Hu Q-H, Chu Z-X, Zhang J, Li Y-Z, Lu L, Wang Z, Fu J-H, Chen $X$. Recreational drug use and risks of HIV and sexually transmitted infections among Chinese men who have sex with men: Mediation through multiple sexual partnerships. BMC Infect Dis. 2014;14(1):642.

33. Wei C, Guadamuz TE, Lim SH, Huang Y, Koe S. Patterns and levels of illicit drug use among men who have sex with men in Asia. Drug Alcohol Depend. 2012;120(1):246-9.

34. Zou H, Hu N, Xin Q, Beck J. HIV testing among men who have sex with men in China: a systematic review and meta-analysis. AIDS Behav. 2012;16(7): 1717-28.

35. Na W, Sun Yanming LH: A Survey on HIV prevalence among Young MSM in Beijing City Chinese Journal of AIDS \& STD 2013, 19(4).

36. Qi X, Xu J, Zhang Z, Li S-m, Xiao D, Gao Y-j, Li D-I, Jiang G-y, Luo F-j, Ruan Y-h et al.: A survey on HIV and Syphilis Infections among MSM population in Beijing. Chinese Preventive Medicine 2013, 14(6).

37. Fu J, Gong Z. An analysis of HIV prevalence and related risk behavior amongst MSM in Jiangxi Province. Jiangxi Med J. 2014;49(10):970-3.

38. Fan EL. HIV testing as prevention among MSM in China: the business of scaling-up. Glob Public Health. 2014;9(1-2):85-97.

39. Liu Y, Sun X, Qian H-Z, Yin L, Yan Z, Wang L, Jiang S, Lu H, Ruan Y, Shao Y. Qualitative Assessment of Barriers and Facilitators of Access to HIV Testing Among Men Who Have Sex with Men in China. AIDS Patient Care STDs. 2015;29(9):481-9.

40. Wei C, Yan H, Yang C, Raymond HF, Li J, Yang H, Zhao J, Huan X, Stall R. Accessing HIV testing and treatment among men who have sex with men in China: a qualitative study. AIDS Care. 2014;26(3):372-8.

41. Wu Z, Zhao Y, Ge X, Mao Y, Tang Z, Shi CX, Chen C, Li Y, Qiu X, Nong G. Simplified HIV Testing and Treatment in China: Analysis of Mortality Rates Before and After a Structural Intervention. PLoS Med. 2015;12(9):e1001874.

42. Tang H, Mao Y, Shi CX, Han J, Wang L, Xu J, Qin Q, Detels R, Wu Z. Baseline CD4 Cell Counts of Newly Diagnosed HIV Cases in China: 2006-2012. PLoS One. 2014;9(6):e96098.

43. Davis A, Best J, Luo J, Van Der Pol B, Dodge B, Meyerson B, Aalsma M, Wei C, Tucker JD: Risk behaviours, HIV/STI testing and HIV/STI prevalence between men who have sex with men and men who have sex with both men and women in China. Int J STD AIDS 2015: 0956462415596302

44. Zhou W, Zhao M, Wang X, Schilling RF, Zhou S, Qiu H-Y, Xie N-H, Liu M-Q, Dong H-S, Yao Z-Z. Treatment Adherence and Health Outcomes in MSM with HIV/AIDS: Patients Enrolled in "One-Stop" and Standard Care Clinics in Wuhan China. PLoS One. 2014;9(12):e113736.

45. Zhang F, Dou Z, Ma Y, Zhang Y, Zhao Y, Zhao D, Zhou S, Bulterys M, Zhu H, Chen RY. Effect of earlier initiation of antiretroviral treatment and increased treatment coverage on HIV-related mortality in China: a national observational cohort study. Lancet Infect Dis. 2011;11(7):516-24.

46. Muessig KE, McLaughlin MM, Nie JM, Cai W, Zheng H, Yang L, Tucker JD. Suboptimal antiretroviral therapy adherence among HIV-infected adults in Guangzhou, China. AIDS Care. 2014;26(8):988-95.

47. Tang W, Best J, Zhang Y, Liu FY, Tso LS, Huang S, Yang B, Wei C, Tucker JD: Gay mobile apps and the evolving virtual risk environment: a cross-sectional online survey among men who have sex with men in China. Sex Transm Infect 2016

48. Christopoulos KA, Das M, Colfax GN. Linkage and retention in HIV care among men who have sex with men in the United States. Clin Infect Dis. 2011;52 suppl 2:S214-22

49. Tang W, Best J, Zhang Y, Liu F, Tso LS, Huang S, Yang B, Wei C, Tucker JD. Gay Mobile Apps as an Emerging Virtual Risk Environment: A Cross-Sectional Online Survey among Men who have Sex with Men in China. Vancover: IAS; 2015.

50. Zheng $L$, Zheng $Y$. Efficacy of human immunodeficiency virus prevention interventions among men who have sex with men in China: a meta-analysis. Sex Transm Dis. 2012;39(11):886-93.

51. China_Food_and_Drug_Adminstration: Database of approved Active Pharmaceutical Ingredients (APIs) and API manufacturers in China. In Beijing; 2015.

52. Tang W, Han L, Best J, Zhang Y, Mollan K, Kim J, Liu F, Hudgens M, Bayus B, TerrisPrestholt F, et al. Crowdsourcing HIV Test Promotion Videos: A Noninferiority Randomized Controlled Trial in China. Clin Infect Dis. 2016;62(11):1436-42.

53. Wang X, Wu Z, Tang Z, Nong Q, Li Y. Promoting HIV testing with home self-test kit among men who have sex with men in China: a feasibility study. Lancet. 2015;386:S68.

54. Zhou Y, Li D, Lu D, Ruan Y, Qi X, Gao G. Prevalence of HIV and syphilis infection among men who have sex with men in China: a meta-analysis. Biomed Res Int. 2014;2014:620431.
55. Yan H, Zhang R, Wei C, Li J, Xu J, Yang H, McFarland W. A peer-led, community-based rapid HIV testing intervention among untested men who have sex with men in China: an operational model for expansion of HIV testing and linkage to care. Sex Transm Infect. 2014;90(5):388-93.

56. WHO: Consolidated Guidelines on HIV Testing Services. In. Geneva; 2015.

57. Zhong F, Tang W, Cheng W, Lin P, Wu Q, Cai Y, Tang S, Fan L, Zhao Y, Chen $X$ et al.: Acceptability and Feasibility of a Social-Entrepreneurship Model to Promote HIV Self-testing and linkage to care among MSM. HIV Med 2016, Accepted, in press.

58. Cheng W, Cai Y, Tang W, Zhong F, Meng G, Gu J, Hao C, Han Z, Li J, Das A, et al. Providing HIV-related services in China for men who have sex with men. Bull World Health Organ. 2016;94(3):222-7.

59. Avery M, Meng G, Mills S. Two internet-based approaches to promoting HIV counselling and testing for MSM in China. Digit Cult Educ. 2014;6:216-29.

60. A New HIV/AIDS Intervention Model for MSM: Experience of Using Social Media for AIDS Interventions and HIV Testing Mobilization in China. ChinaGates Foundation HIVPrevention Cooperation Program. September 2013. http://ncaids.chinacdc.cn/zyxz/zlk/201401/W020140107512046173249.pdf. Accessed 7 Oct 2016.

61. Tso LS, Tang W, Li H, Yan HY, Tucker JD. Social media interventions to prevent HIV: a review of interventions and methodological considerations. Curr Opin Psychol. 2016;9:6-10.

62. Guo B, Yu Z, Zhou X, Zhang D: From participatory sensing to mobile crowd sensing. In: Pervasive Computing and Communications Workshops (PERCOM Workshops), 2014 IEEE International Conference on: 2014: IEEE; 2014: 593-598.

63. Tang W, Mao J, Liu C, Mollan K, Li H, Wong T, Zhang Y, Tang S, Hudgens M, Qin Y, et al. Reimagining Health Communication: A Non-Inferiority Randomized Controlled Trial of Crowdsourcing in China. Durban: International AIDS Society; 2016.

64. WHO: Guidance on oral pre-exposure prophylaxis (PrEP) for serodiscordant couples, men and transgender women who have sex with men at high risk of HIV. In. Geneva; 2012. http://www.who.int/hiv/pub/guidance_prep/en/. Accessed 17 Oct 2016

65. Jackson T, Huang A, Chen H, Gao X, Zhong X, Zhang Y. Cognitive, psychosocial, and sociodemographic predictors of willingness to use HIV pre-exposure prophylaxis among Chinese men who have sex with men. AIDS Behav. 2012;16(7):1853-61.

66. Mijiti P, Yahepu D, Zhong X, Sun Y, Zhao T, Zhao Z, Abuduaili Z, Zhou H, Meng $F$, Dai J. Awareness of and willingness to use oral pre-exposure prophylaxis for HIV prevention among HIV-serodiscordant heterosexual couples: a cross-sectional survey in Xinjiang, China. PLoS One. 2013;8(7):e67392.

67. Zhang Y, Peng B, She Y, Liang H, Peng H-B, Qian H-Z, Vermund SH, Zhong X-N, Huang A. Attitudes toward HIV pre-exposure prophylaxis among men who have sex with men in western China. AIDS Patient Care STDs. 2013;27(3):137-41.

68. Zhou F, Gao L, Li S, Li D, Zhang L, Fan W, Yang X, Yu M, Xiao D, Yan L. Willingness to accept HIV pre-exposure prophylaxis among Chinese men who have sex with men. PLoS One. 2012;7(3):e32329.

69. Gao F, Zhong X, Peng B, Zhang Y, Liang H, Zou Y, Dai J, Liu Q, Song B, Huang W, et al. Pre-exposure prophylaxis for HIV high risk groups: analysis of clinical compliance. Acad J Second Mil Med Uni. 2012;33(11):1186-93.

70. Zhang $Y$, Zhong $X$, Peng B, Huang W, Huang A. Cohort retention and predictors in HIV pre exposure prophylaxis clinical trial among men who have sex with men. Acad J Third Mil Med Uni. 2013;35(22):3.

71. Tucker JD, Fenton KA, Peckham R, Peeling RW. Social entrepreneurship for sexual health (SESH): a new approach for enabling delivery of sexual health services among most-at-risk populations. PLoS Med. 2012;9(7):e1001266.

72. Taggart T, Grewe ME, Conserve DF, Gliwa C, Roman Isler M. Social Media and HIV: A Systematic Review of Uses of Social Media in HIV Communication. J Med Internet Res. 2015;17(11):e248.

73. Tucker JD. Hepatitis Testing Innovation Contest. Barcelona: International Liver Congress-EASL; 2016.

74. McNairy ML, Howard AA, El-Sadr WM. Antiretroviral therapy for prevention of HIV and tuberculosis: a promising intervention but not a panacea. J Acquir Immune Defic Syndr. 2013;63:S200-7.

75. Peters DH, Tran NT, Adam T. Implementation Research in Health: A Practical Guide. Geneva: World Health Organization; 2013.

76. Zhang Y, Kim JA, Liu F, Tso LS, Tang W, Wei C, Bayus BL, Tucker JD. Creative Contributory Contests to Spur Innovation in Sexual Health: 2 Cases and a Guide for Implementation. Sex Transm Dis. 2015;42(11):625-8. 